\title{
El castellano de la comunidad shipibo de Cantagallo: estudio acústico de la duración vocálica
}

\section{The Spanish of the Cantagallo Shipibo Community: An Acoustic Study of Vowel Duration}

\author{
Paloma Pinillos Chávez \\ The Ohio State University, Columbus, Estados Unidos \\ https://orcid.org/oooo-0003-2504-1135 \\ pinilloschavez.1@osu.edu
}

\begin{abstract}
Resumen
Este estudio explora la duración vocálica en el castellano hablado por bilingües shipibo-castellano de la comunidad de Cantagallo. Este análisis se realiza tomando en cuenta el trabajo de Elías Ulloa (2011) quien examina las vocales largas del shipibo a partir de la noción de tamaño mínimo de palabra, requerimiento que no está presente en castellano. Los resultados indican que los hablantes bilingües producen vocales con mayor duración que las de los monolingües. Además, la duración vocálica entre estos bilingües varía dependiendo de su dominancia hacia shipibo o castellano. Por otro lado, sugiero que la naturaleza de las vocales largas producidas en el castellano de los hablantes shipibo podría deberse a la transferencia del requerimiento del peso silábico del shipibo al castellano.
\end{abstract}

Palabras clave: contacto de lenguas, bilingüismo, fonología, fonética

\begin{abstract}
This study explores the vowel duration spoken by Shipibo-Spanish bilinguals from the community of Cantagallo. This research takes into account the work of Elías Ulloa (2011), who analyzes the duration of Shipibo vowels founded on the notion of minimum word size, requirement that doesn't exist in Spanish. Results indicate that bilingual speakers produce longer vowels than monolingual speakers. Also, vowel duration among these bilinguals varies depending on their Shipibo and Spanish dominance. On the other hand, I suggest that the nature of the long vowels produced in Spanish by Shipibo speakers could be due to the transfer of the requirement of syllabic weight from Shipibo to Spanish.
\end{abstract}

Keywords: language contact, bilingualism, phonology, phonetics 
El castellano de la comunidad shipibo de Cantagallo: estudio acústico de la...

Paloma Pinillos Chávez

\section{Introducción}

La comunidad shipiba es originaria de la Amazonía peruana, específicamente del departamento de Ucayali. En la actualidad también se encuentran comunidades en los departamentos de Madre de Dios, Loreto y Huánuco. La población estimada es de 32964 habitantes, número que los ubica entre los grupos indígenas con mayor población en la Amazonía peruana (INEI, 2007; Ministerio de Cultura del Perú, 2019). Entre los años 1980 y 2001, se dieron movimientos migratorios constantes de la comunidad shipiba hacia Lima, la capital. En el año 2001, tras una migración masiva debido a la inestabilidad económica en la Amazonía, fundaron la comunidad de Cantagallo en Barrios Altos, la cual, posteriormente, se movilizó y estableció en el distrito de El Rímac (Anzualdo, 2015).

A pesar de que esta comunidad se encuentra físicamente lejos de la Amazonía y vive en una zona urbana, sus miembros mantienen su identidad y lengua (Elías Ulloa, 2011). En la comunidad se habla shipibo y castellano, pero la elección de la lengua varía dependiendo si se encuentran dentro o fuera de la comunidad (Elías Ulloa, 2011). Casi todos los miembros de generaciones medias y mayores hablan shipibo como primera lengua y castellano como segunda lengua. Sin embargo, su dominio de castellano varía dependiendo de factores como edad, género y nivel de contacto con las áreas urbanas (Adelaar et al., 2011). Por otro lado, los hablantes shipibo más jóvenes muestran evidencia de préstamo sintáctico, morfológico, semántico y fonológico del castellano al shipibo (Adelaar et al., 2011).

El presente estudio investiga el contacto lingüístico entre el shipibo y el castellano en la comunidad de Cantagallo. En específico, estudio la influencia de los patrones de duración vocálica del shipibo en la duración de las vocales del castellano de los hablantes bilingües shipibo-castellano de Cantagallo. Para analizar esta influencia, me baso en lo propuesto por Elías Ulloa (2011) quien, tras analizar la naturaleza de las vocales largas del shipibo, sugiere que esta duración vocálica parte del requerimiento de un tamaño mínimo de palabra. Este investigador señala que este requerimiento explica la naturaleza de las vocales largas tanto en los monosílabos nominales del shipibo como en sus respectivos paradigmas derivados. Basada en esto, los objetivos de este estudio son, en primer lugar, analizar el comportamiento de las vocales castellanas de los hablantes bilingües shipibo-castellano con el fin de examinar una posible transferencia de los patrones de duración vocálica del shipibo al castellano y, en segundo lugar, comparar y analizar la duración de las vocales según el nivel de dominancia lingüística del shipibo y del castellano de estos hablantes bilingües. 


\section{Literatura previa}

\subsection{Duración vocálica en castellano}

Navarro Tomás es uno de los pioneros en el estudio de la duración de las vocales del castellano $(1916,1917)$. En sus investigaciones encuentra tres fenómenos importantes en las vocales castellanas: (1) son más cortas en comparación con otros idiomas como el inglés y el alemán, (2) la duración de las vocales tiene un correlato articulatorio, es decir, las vocales más altas /i, u/ son más cortas que la vocal baja /a/ y (3) las vocales son más cortas en sílabas cerradas que en sílabas abiertas. Por otro lado, Quilis y Esgueva (1983) muestran que las vocales castellanas varían según el dialecto. Estos autores centran su estudio en la comparación del español de América y España, y concluyen que los hablantes de castellano americano presentan una duración vocálica mayor que los hablantes de España.

Estudios más recientes continúan corroborando estos resultados. Morrison y Escudero (2007) realizaron comparaciones dialectales entre las vocales del castellano hablado en Lima, Perú, y Madrid, España, para explorar la duración vocálica en ambas variedades. Los resultados muestran que no hay diferencia entre los valores formánticos en estos dos dialectos. Sin embargo, encuentran diferencias significativas en la duración y la frecuencia fundamental. En cuanto a la duración, los hablantes de castellano peruano producen vocales más largas que los de España: la duración media de la vocal de los españoles fue de $69 \mathrm{~ms}$., mientras que la media de los hablantes peruanos fue de 98 ms., es decir, 28 ms. (33.9 \%) más larga. Por otro lado, la frecuencia fundamental de los hablantes peruanos resulta más alta $(149 \mathrm{~Hz})$ en comparación con los hablantes españoles $(136 \mathrm{~Hz})$. Por su parte, Chládková et al. (2011) examinan también estos dos dialectos y muestran que las vocales del castellano peruano son un poco más largas que las vocales de los españoles. Además, encuentran que las palabras aisladas tienden a tener vocales más largas que dentro de una frase portadora para ambos dialectos. Sin embargo, los resultados no fueron estadísticamente significativos.

En cuanto al estudio de la duración vocálica de dialectos del Perú, García (2016) analiza la duración de las vocales en el castellano peruano de Lima y Pucallpa bajo la hipótesis de que las vocales del castellano de Pucallpa son más largas que las vocales del castellano de Lima. Sus resultados muestran que existen diferencias significativas entre la duración de las vocales de estos dos dialectos. García explica que en Pucallpa las vocales en castellano son más largas tanto en posición tónica como no tónica en comparación con el castellano de Lima. Además, 
El castellano de la comunidad shipibo de Cantagallo: estudio acústico de la...

Paloma Pinillos Chávez

en el castellano de Pucallpa, la vocal post-tónica tiene una duración menor que la pre-tónica. Por el contrario, en el castellano de Lima, las vocales pretónicas y postónicas tienen la misma duración, mientras que la tónica es un poco más larga que la pretónica y la postónica (García, 2016). Así, en el dialecto amazónico las vocales son fonéticamente más largas en comparación con el dialecto limeño, lo que sustenta estudios previos (Saldaña Fernández, 2009) que indican que en la variedad amazónica hay un alargamiento de vocales considerable.

\subsection{Duración vocálica en shipibo}

El shipibo y el castellano son lenguas que vienen de dos familias lingüísticas no relacionadas (pano y romance, respectivamente) y muestran características lingüísticas diferentes. En cuanto a su sistema vocálico, estas lenguas se diferencian en cantidad y calidad. Por un lado, el castellano cuenta con un sistema pentavocálico, /i, e, a, o, u/; mientras que el Shipibo tiene vocales cortas y largas: /i/ /i / / / /a/ y, su contraparte larga, /i:/, /ì:/, / :/ /a:/ (Elías-Ulloa, 2006). De acuerdo con Elías-Ulloa (2011), las vocales largas son aproximadamente $80 \mathrm{~ms}$. más largas que las vocales cortas. Además, las palabras monosilábicas presentan vocales con una duración mayor de entre 20 y 45 ms. en comparación con las palabras bisilábicas y trisilábicas, las cuales presentan una duración vocálica virtualmente igual.

En cuanto a la fonología del shipibo, Elías-Ulloa (2011) señala que la duración vocálica en esta lengua se puede explicar a partir del requerimiento del tamaño mínimo de palabra. Según este autor, cuando una raíz monosilábica no tiene sufijo siempre aparecerá con una vocal larga debido al requerimiento de un mínimo de dos moras. Así, el peso silábico sería un requerimiento en esta lengua: de tener una raíz monosilábica con una vocal corta, solo se tendría una mora, lo que infringiría la restricción del tamaño mínimo de palabra. Los siguientes ejemplos son casos de monosílabos sustantivos del shipibo:

(1) a. [kv:] 'pus'

b. [t $\mathrm{fi}$ :] 'fuego'

Nota: Ejemplos tomados de Elías-Ulloa (2011, pp. 242-243)

Por otro lado, las raíces verbales monosilábicas en esta lengua funcionan de manera diferente, ya que, a diferencia de las raíces monosilábicas nominales, las verbales deben siempre tener un sufijo. Al siempre estar esta raíz anexada a un sufijo, cumple con el requerimiento del tamaño mínimo de palabra y, por tanto,

$170 \quad$ Lengua y Sociedad. Revista de Lingüística Teórica y Aplicada 
no necesita de una vocal larga, dos moras, para compensar el peso silábico. En (2) se puede observar algunos de estos casos:

(2) a. ['a.ti] 'hacer'

b. ['ni.ti] 'caminar'

c. ['hu.ti] 'venir'

Nota: Ejemplos tomados de Elías-Ulloa (2011, p. 242)

Ahora bien, lo interesante de las raíces nominales monosilábicas del shipibo es la herencia de sus vocales largas en sus paradigmas (Elías-Ulloa, 2011). En la Tabla 1, la raíz nominal monosilábica [kv:] 'pus' se convierte en el verbo ['ku: ti] 'supurar' cuando se agrega el sufijo infinitivo /-ti/ y mantiene la vocal larga de la raíz nominal a pesar de que ya se está satisfaciendo el tamaño mínimo de la palabra. La preservación de esta vocal larga ocurre en todas las palabras del paradigma de la raíz [kv:], como se puede ver en los otros ejemplos de la Tabla 1.

Tabla 1. Raíz nominal monosilábica [kv:] y tres de sus paradigmas verbales heredando la vocal larga

\begin{tabular}{l|l|l}
\hline $\begin{array}{l}\text { Raíz nominal } \\
\text { monosilábica }\end{array}$ & Sufijos & Paradigma verbal \\
\hline \multirow{3}{*}{$\mathrm{kv}:$ ' $p u s '$} & /-ti/ infinitivo & ['kv:ti] 'supurar' \\
\cline { 2 - 3 } & /-ki/ aspecto completivo & ['kv:.ki] 'supuró' \\
\cline { 2 - 3 } & /-wan-/ pasado reciente & ['kv:.wan.ki] 'supuró hoy día' \\
\hline
\end{tabular}

Fuente. Adaptado de Elías-Ulloa (2011, p. 242).

Esta preservación de la duración vocálica en el nivel paradigmático no es exclusiva del paradigma verbal, sino que también ocurre en el paradigma nominal. En la Tabla 2, se puede ver el ejemplo de la raíz nominal monosilábica [t $\mathrm{ji}$ :] 'fuego' que también les hereda la vocal larga a los sustantivos formados tras agregarles los sufijos /-ki/ y /- $\widehat{d} z_{i} /$. 
El castellano de la comunidad shipibo de Cantagallo: estudio acústico de la...

Paloma Pinillos Chávez

Tabla 2. Raíz nominal monosilábica [t $f$ i:] y dos de sus paradigmas nominales heredando la vocal larga

\begin{tabular}{|c|c|c|}
\hline Raíz nominal monosilábica & Sufijos & Paradigma nominal \\
\hline \multirow{2}{*}{ [tfi:] 'fuego' } & /-ki/ locativo & ['t $\mathrm{f} \mathrm{i}: \mathrm{ki}]$ 'fuego en un lugar específico' \\
\hline & 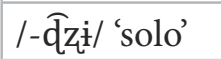 & ['t $\mathrm{ti}: . \overline{d z i}]$ 'solo el fuego' \\
\hline
\end{tabular}

Fuente. Adaptado de Elías-Ulloa (2011, p. 243).

A partir de estos datos, Elías-Ulloa (2011) sugiere que la raíz nominal monosilábica en shipibo es la fuente principal de las vocales largas. Basándome en la fonología del shipibo y en lo propuesto por Elías Ulloa, desarrollo un estudio experimental en el que analizo las duraciones vocálicas del castellano hablado por hablantes bilingües shipibo-castellano de la comunidad shipiba de Cantagallo.

\section{El presente estudio}

El propósito en este estudio es examinar el contacto lingüístico entre el shipibo y el castellano en la comunidad de Cantagallo. En específico, analizo la influencia de los patrones de la duración vocálica del shipibo en el castellano hablando por bilingües shipibo-castellano. Con el fin de examinar lo propuesto, las preguntas de investigación planteadas en este estudio son las siguientes:

1. ¿Los patrones de duración del shipibo han influenciado la duración vocálica del castellano de los hablantes bilingües shipibo-castellano?

2. ¿La duración vocálica de estos hablantes bilingües varía dependiendo de su nivel de bilingüismo?

2.1 ¿Qué diferencias existen en la duración de las vocales del castellano a partir de la dominancia lingüística hacia el shipibo o hacia el castellano?

2.2 ¿Los hablantes más dominantes en shipibo producen vocales con mayor duración en comparación a los más dominantes en castellano? 


\section{Metodología}

\subsection{Participantes}

Seis (6) bilingües shipibo-castellano de la comunidad de Cantagallo participaron en el presente estudio. De estos, cuatro fueron mujeres y dos hombres con una media de edad de 33.5 años al momento del estudio (rango de edad de 18 a 61 años). Todos los participantes nacieron en Ucayali, fueron criados en shipibo, residen en la comunidad de Cantagallo y hablan exclusivamente shipibo en casa y en la comunidad. Para fines comparativos, también conté con un grupo control. Este estuvo compuesto por seis (6) hablantes monolingües de castellano: cuatro mujeres y dos hombres con una edad media de 36 (rango de edad de 26 a 56 años). Todos los del grupo control fueron criados exclusivamente en castellano, ninguno tenía conocimientos de shipibo o fue criado como hablante bilingüe.

Para determinar el nivel de bilingüismo de los participantes shipibo, utilicé el Bilingual Language Profile (BLP) (Birdsong, D. et al, 2012). El cuestionario de BLP tiene como objetivo medir el grado de dominio de las lenguas de los participantes y, así, ubicarlos en un continuo de bilingüismo. Este cuestionario tiene cuatro módulos en los que se obtiene información específica sobre el participante en relación con su (1) historial lingüístico, (2) uso de la lengua, (3) dominio de la lengua y (4) actitudes lingüísticas. Luego de completar el cuestionario, los participantes pueden obtener una puntuación máxima o mínima de +218 o -218. Los puntajes positivos indican dominio hacia una lengua, mientras que los puntajes negativos indican dominio hacia la otra lengua. Cualquiera de los dos idiomas puede ubicarse en el extremo positivo o negativo de este continuo. En este estudio, los puntajes positivos indican dominio en castellano; mientras que los puntajes negativos, dominio en shipibo. 
El castellano de la comunidad shipibo de Cantagallo: estudio acústico de la...

Paloma Pinillos Chávez

Tabla 3. Información demográfica, educacional y de BLP de los hablantes bilingües shipibo-castellano

\begin{tabular}{l|l|l|l|l|l|l|}
\hline Hablantes & Etiqueta & Edad & Género & Educación & $\begin{array}{l}\text { Lenguas } \\
\text { de educación }\end{array}$ & $\begin{array}{l}\text { Puntaje } \\
\text { BLP }\end{array}$ \\
\hline Participante 1 & $\mathrm{P}(18)$ & 18 & $\mathrm{~F}$ & $\begin{array}{l}\text { Sec. } \\
\text { incompleta }\end{array}$ & $\begin{array}{l}\text { Prim. y sec. en } \\
\text { castellano (11 años) }\end{array}$ & 52.766 \\
\hline Participante 2 & $\mathrm{P}(28)$ & 28 & $\mathrm{M}$ & $\begin{array}{l}\text { Univ. } \\
\text { incompleta }\end{array}$ & $\begin{array}{l}\text { Prim. y sec. bilingüe } \\
\text { (12 años) y univ. en } \\
\text { castellano (6 años) }\end{array}$ & -40.5 \\
\hline Participante 3 & $\mathrm{P}(29)$ & 29 & $\mathrm{~F}$ & $\begin{array}{l}\text { Univ. } \\
\text { completa }\end{array}$ & $\begin{array}{l}\text { Prim. y sec. bilingüe } \\
\text { (12 años) y univ. en } \\
\text { castellano (4 años) }\end{array}$ & -94.62 \\
\hline Participante 4 & $\mathrm{P}(32)$ & 32 & $\mathrm{~F}$ & $\begin{array}{l}\text { Sec. } \\
\text { completa }\end{array}$ & $\begin{array}{l}\text { Prim. y sec. bilingüe } \\
\text { (12 años) }\end{array}$ & -49.85 \\
\hline Participante 5 & $\mathrm{P}(33)$ & 33 & $\mathrm{~F}$ & $\begin{array}{l}\text { Sec. } \\
\text { incompleta }\end{array}$ & $\begin{array}{l}\text { Prim. y sec. bilingüe } \\
\text { (10 años) }\end{array}$ & -65.924 \\
\hline Participante 6 & $\mathrm{P}(61)$ & 61 & $\mathrm{M}$ & $\begin{array}{l}\text { Sec. } \\
\text { completa }\end{array}$ & $\begin{array}{l}\text { Prim. y sec. bilingüe } \\
\text { (12 años) }\end{array}$ & -61.384 \\
\hline
\end{tabular}

La Tabla 3 presenta la información completa de los hablantes bilingües shipibo-castellano. En esta, resumo la información demográfica y educacional de estos bilingües, así como sus puntajes del BLP. Como muestra la tabla, de estos seis participantes bilingües, solo una recibió educación primaria y secundaria exclusivamente en castellano, mientras que los otros cinco recibieron educación en ambas lenguas, shipibo y castellano. En cuanto a nivel de educación, cuatro tienen educación secundaria completa o incompleta, y dos tienen estudios universitarios completos o parciales. En cuanto a los resultados del BLP, cinco de seis participantes obtuvieron puntajes negativos, lo cual indica que tiene mayor dominancia del shipibo que del castellano. Estos participantes son los que coincidentemente registran más de diez años de educación bilingüe. La participante más joven del grupo es la única que obtuvo puntaje positivo, lo cual indica más mayor dominancia en castellano.

En este estudio, solo los bilingües shipibo-castellano completaron este cuestionario. Cada uno de los hablantes bilingües llenó el cuestionario en castellano guiados por mi. Primero, leí a los participantes cada una de las preguntas del cuestionario sección por sección y llené cada una de las respuestas a mano en una versión en papel y lápiz. También grabé en audio todo el proceso del cuestionario 
para luego corroborar cada respuesta. Además, debido a que no existe un cuestionario castellano-shipibo, adapté la versión español-catalán (Birdsong, D. et al, 2012) para crear una versión apropiada para este estudio.

\subsection{Recolección de datos}

Luego de completar el cuestionario de BLP, los participantes realizaron una tarea de lectura. En esta tarea, les pedí a los participantes que leyeran en total cincuenta y tres (53) oraciones en castellano. Cuarenta (40) de estas oraciones contenían las palabras de interés para este estudio: monosílabos nominales y sus respectivos paradigmas nominales. Cada una de estas oraciones contenía solo una de las palabras de interés, la cual se colocó en posición media de la oración. Las vocales sujetas a análisis fueron las cinco del castellano y todas se encontraron en sílaba tónica. Además, incorporé trece (13) oraciones extra como distractores. Estas oraciones no contaban con monosílabos ni derivados de monosílabos. En la Tabla 4, se pueden observar ejemplos de las oraciones con las palabras de interés que utilicé en este estudio.

Tabla 4. Muestra de monosílabos nominales y sus paradigmas nominales utilizados para la tarea de lectura

\begin{tabular}{l|l}
\hline Raíces monosilábicas nominales & Paradigmas nominales \\
\hline Ella come pan todos los días & Ella come panes todos los días \\
\hline Hay un pez en el lago & Hay unos peces en el lago \\
\hline Yo tengo mil cosas allá & Yo tengo miles de cosas \\
\hline Falta un sol para comprar & Faltan cinco soles para comprar \\
\hline Se pasó una luz verde ayer & Se pasó unas luces verdes ayer \\
\hline
\end{tabular}

Nota: Las vocales analizadas están subrayadas.

El procedimiento de grabación de estas oraciones se llevó de la siguiente manera. Primero, les expliqué a los participantes paso a paso en qué consistía esta tarea de lectura. Les mostré la lista impresa con las cuarenta y tres oraciones en castellano, y les expliqué que tendrían que leer cada una de estas oraciones en castellano. Antes de iniciar la grabación, cada uno de los participantes revisó la lista, la cual contenía las oraciones en un orden aleatorio. Luego de que el participante 
El castellano de la comunidad shipibo de Cantagallo: estudio acústico de la...

Paloma Pinillos Chávez

terminó de revisar la lista de oraciones y de responder dudas sobre las oraciones, procedimos con la tarea de lectura. Para controlar la velocidad en la lectura, les expliqué a los participantes que debían leer las oraciones con ritmo natural, ni muy rápido, ni muy lento. Además, les fui señalando cada una de las oraciones para evitar transiciones muy rápidas de oración en oración. Una vez terminada la tarea de lectura, les di una pausa a cada uno de los participantes y luego se procedió a volver a leer la lista de oraciones en su totalidad. De esta forma, cada participante leyó un total de ciento seis (106) oraciones para esta tarea de lectura (80 oraciones con las palabras de interés y 26 oraciones distractoras).

Las grabaciones de los hablantes bilingües shipibo-castellano se realizaron en la comunidad de Cantagallo en cada una de las viviendas de los participantes. En el caso de la grabación del grupo control, esta se realizó en las viviendas de los participantes, localizadas en la zona de Lima centro. La grabadora que utilicé para ambas grabaciones fue una Olympus modelo Linear PCM recorder LS-11 y los audios se grabaron en formato WAV.

\subsection{Análisis de datos}

Para el análisis de datos, primero segmenté cada una de las vocales de interés de los monosílabos y los paradigmas usando el programa Praat (Boersma y Weenink, 2015). Siguiendo investigaciones previas (Guion, 2003; O’Rourke, 2010; ElíasUlloa, 2011; y Amengual, 2011 y 2016), consideré el comienzo y el final de F1 y F2 para marcar el comienzo y el final de la vocal. Una vez segmentadas las vocales, medí su duración. También consideré las formas de la onda en la medición de la duración siguiendo a Abramson y Reo (1990). Además, siguiendo a García (2016), normalicé las medidas de las vocales para controlar las posibles diferencias en la velocidad del habla. De esta forma, obtuve la duración normalizada de cada vocal haciendo una división entre la duración de la vocal y la duración de la oración que contenía la vocal.

En total, recogí 480 estímulos para cada uno de los grupos de participantes (sin considerar los distractores). De este total de estímulos, analicé 443 del grupo de hablantes bilingües (entre 71 y 76 estímulos por participante) y 464 del grupo de control (entre 75 y 79 estímulos por participante). No consideré la cantidad restante de palabras de interés debido a interferencia en los audios o pronunciación inadecuada de las palabras. Además, para mostrar que una mayor duración en las vocales no es una propiedad general en las vocales del castellano de estos

176 Lengua y Sociedad. Revista de Lingüística Teórica y Aplicada 
bilingües shipibo-castellano, también segmenté y medí las vocales de palabras en donde no se espera una vocal larga. Así, escogí 40 sustantivos bisilábicos de los audios de cada uno de los seis hablantes bilingües para el análisis (240 estímulos en total). Estos sustantivos no fueron derivados de monosílabos y la vocal analizada fue la tónica.

En cuanto al análisis estadístico, utilicé pruebas $t(t$-test) para estimar el nivel de significancia de las medidas de duración de las vocales. La prueba t compara el promedio de dos grupos y establece si la diferencia entre estos grupos es significativa o no. De esta forma, comparé las medidas de duración vocálica de la siguiente manera: (1) vocales de monosílabos de hablantes bilingües shipibo-castellano vs. vocales de monosílabos de hablantes monolingües de castellano; (2) vocales de monosílabos vs. vocales de paradigmas de hablantes bilingües shipibo-castellano; (3) vocales de monosílabos vs. vocales de bisílabos de hablantes bilingües shipibo-castellano; y (4) vocales de bisílabos de hablantes bilingües shipibo-castellano vs. vocales de monosílabos de hablantes monolingües de castellano.

\section{Resultados}

En cuanto a las vocales de monosílabos producidas por los hablantes bilingües y por los hablantes monolingües (grupo control), los bilingües produjeron vocales con mayor duración que los monolingües. En la Figura 1, se puede ver que la vocal con menor duración de los bilingües ([i] con 59.5 ms.) tiene mayor duración que la vocal con mayor duración de los monolingües ([a] con 54.48 ms.). Por otra parte, el continuo de duración vocálica de mayor a menor duración para los hablantes bilingües es $[\mathrm{e}]>[\mathrm{a}]>[\mathrm{o}]>[\mathrm{u}]>[\mathrm{i}]$; mientras que para los hablantes bilingües es $[\mathrm{a}]>[\mathrm{o}]>[\mathrm{u}]>[\mathrm{i}]>[\mathrm{e}]$. Asimismo, los resultados de la prueba $\mathrm{t}$ indican que hay una diferencia significativa al comparar la duración vocálica de los monosílabos de ambos grupos en las cinco vocales castellanas $(\mathrm{p} \leq 0.5)$. 
El castellano de la comunidad shipibo de Cantagallo: estudio acústico de la...

Paloma Pinillos Chávez

Figura 1. Duraciones vocálicas normalizadas de los monosílabos de los hablantes bilingües shipibo-castellano y de los monolingües de castellano

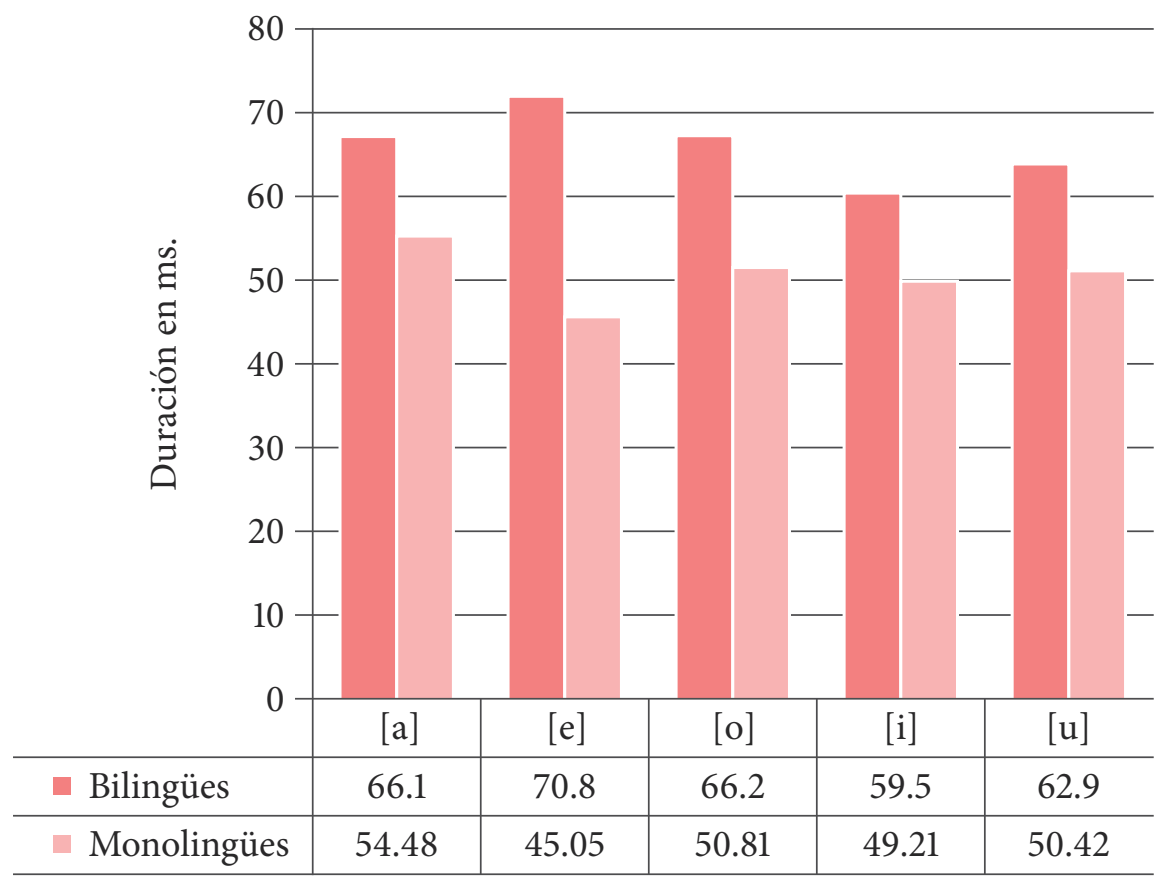

Tras comparar la duración de las vocales de los monosílabos con las duraciones de las vocales de los paradigmas de los hablantes bilingües shipibo-castellano, los resultados muestran que las duraciones son virtualmente las mismas en las cinco vocales. Como se puede observar en la Figura 2, la mayor diferencia de duración vocálica entre monosílabo y paradigma es de $1.9 \mathrm{~ms}$. para la vocal [e], mientras que la menor diferencia es de $0.6 \mathrm{~ms}$. para la vocal [i]. Los resultados de la prueba t señalan que no hay una diferencia significativa al comparar la duración vocálica de los monosílabos y los paradigmas en las cinco vocales castellanas $(p \geq 0.5)$. 
Figura 2. Duraciones vocálicas normalizadas de los monosílabos y los paradigmas de los hablantes bilingües shipibo-castellano

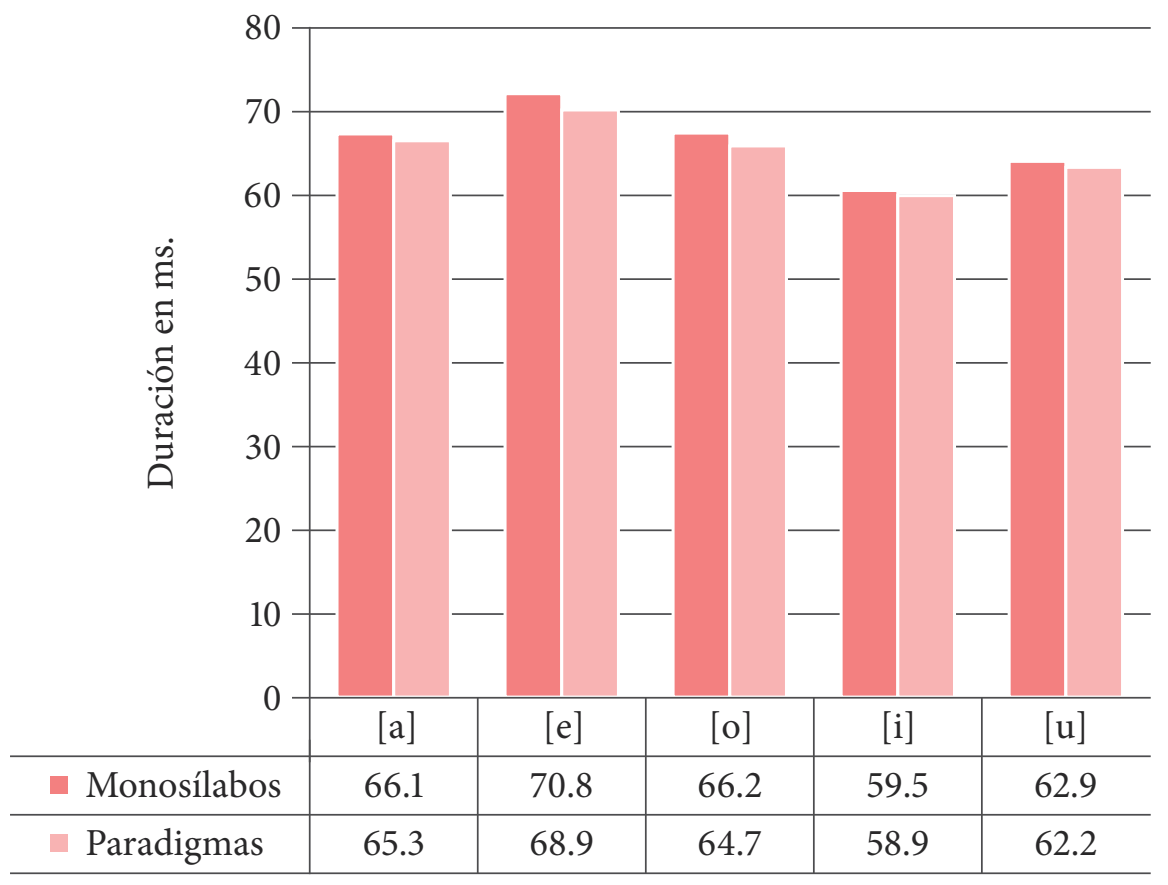

Por otro lado, al comparar la duración de las vocales de los monosílabos con la duración de las vocales de los bisílabos de los hablantes bilingües shipibo-castellano, los resultados indican que la duración vocálica en los monosílabos es mayor que en los bisílabos. En la Figura 3, se puede observar que la vocal con menor duración de los monosílabos ([i] con $59.5 \mathrm{~ms}$.) es mayor que la vocal con mayor duración de los bisílabos ([a] con $51.23 \mathrm{~ms}$.). Asimismo, la mayor diferencia de duración entre monosílabo y bisílabo se da en la vocal [e] con una diferencia de $21.72 \mathrm{~ms}$., mientras que la menor diferencia de duración es de $12 \mathrm{~ms}$. en la vocal [i]. Los resultados de la prueba t señalan que hay una diferencia significativa al comparar la duración vocálica de los monosílabos y los bisílabos en las cinco vocales castellanas $(p \leq 0.5)$. 
El castellano de la comunidad shipibo de Cantagallo: estudio acústico de la...

Paloma Pinillos Chávez

Figura 3. Duraciones vocálicas normalizadas de los monosílabos y los bisílabos de los hablantes bilingües shipibo-castellano

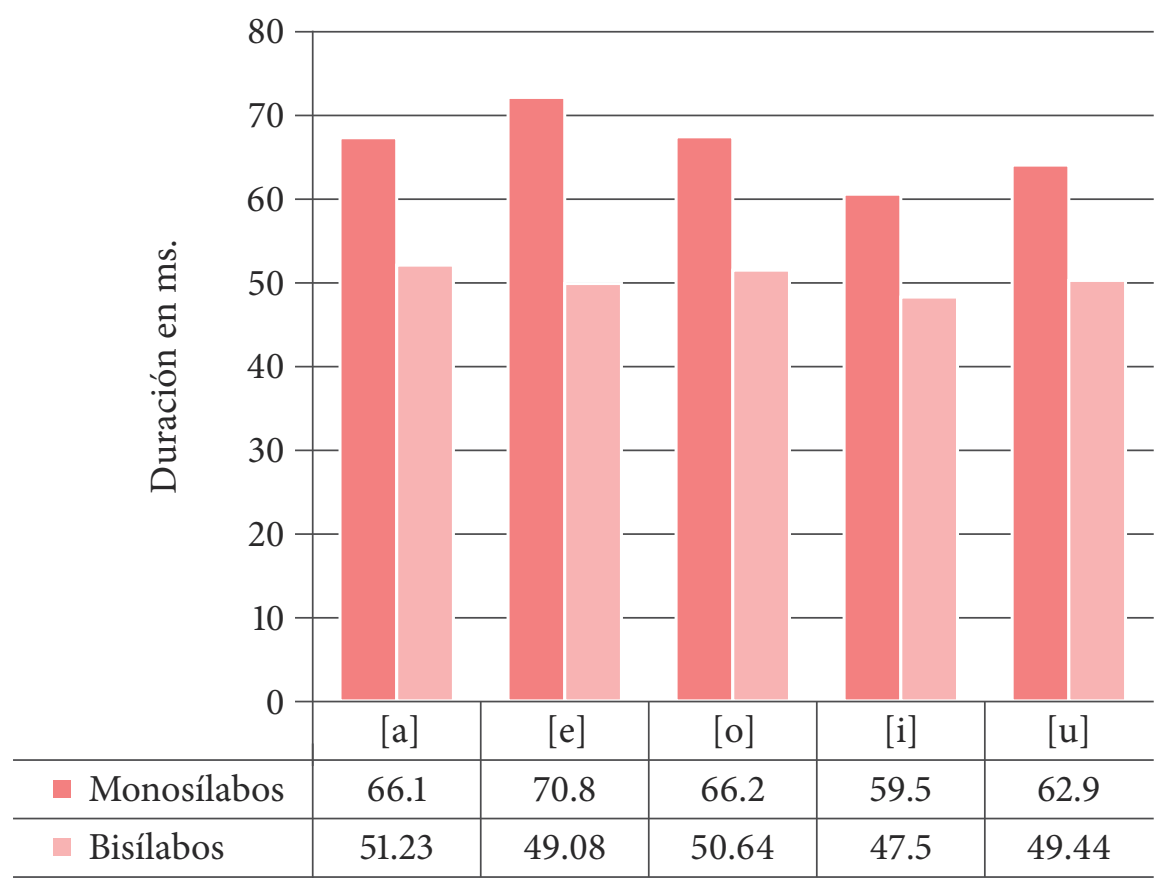

Finalmente, al comparar las vocales de los bisílabos producidos por los hablantes bilingües shipibo-castellano con las vocales de los monosílabos de hablantes monolingües de castellano, los resultados muestran que las duraciones en ambos grupos es virtualmente la misma. Como se puede observar en la Figura 4, la mayor diferencia de duración vocálica entre monosílabo y bisílabo es de $4.03 \mathrm{~ms}$. al comparar las duraciones de la vocal [e], mientras que la menor diferencia es de o.17 ms. al comparar las duraciones de la vocal [o]. Además, la vocal con mayor duración en bisílabo de los bilingües fue [a] con $51.23 \mathrm{~ms}$; mientras que la de mayor duración en monosílabo de los monolingües fue también [a] pero con $54.48 \mathrm{~ms}$. Por otro lado, los resultados de la prueba $t$ indican que no hay una diferencia significativa al comparar la duración vocálica de los monosílabos de los monolingües con los bisílabos de los bilingües en las cinco vocales castellanas $(p \geq 0.5)$. 
El castellano de la comunidad shipibo de Cantagallo: estudio acústico de la...

Paloma Pinillos Chávez

Figura 4. Duraciones vocálicas normalizadas de los bisílabos de los hablantes bilingües shipibo-castellano y de los monosílabos de los monolingües de castellano

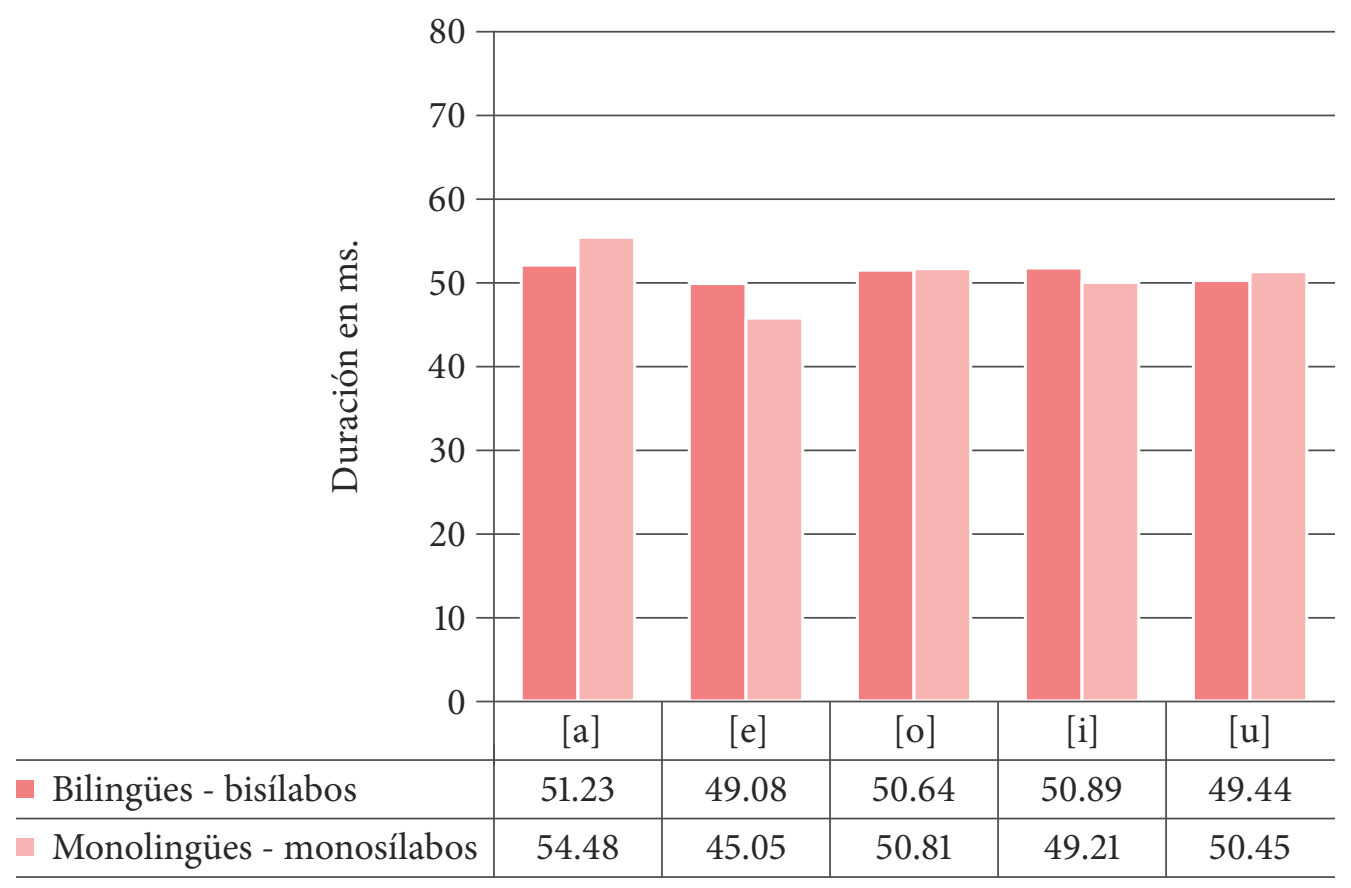

Ahora bien, basada en las duraciones vocálicas de los monosílabos de los hablantes bilingües y en la comparación de estas duraciones con el grupo control, creé tres subgrupos. El primer subgrupo consiste en un hablante bilingüe $\mathrm{P}(28)$ cuyas duraciones vocálicas no mostraron una diferencia significativa con las del grupo de control ( $p \geq 0.5)$. El segundo subgrupo consiste en dos hablantes bilingües, $\mathrm{P}(18)$ y $\mathrm{P}(29)$, y se caracterizan por producir al menos una vocal con duración significativamente mayor que las producidas por el grupo de control. En este caso, ambos hablantes producen la vocal [e] con una duración significativamente mayor $(p \leq 0.5)$ y $\mathrm{P}(18)$ también lo hace con la vocal [o] $(p \leq 0.5)$. Las otras cuatro vocales no mostraron una diferencia significativa al comparar su duración con las del grupo control ( $p \geq 0.5)$. Finalmente, los hablantes del subgrupo tres, $\mathrm{P}(32)$, $\mathrm{P}(33)$ y $\mathrm{P}(61)$, producen todas las vocales con una duración significativamente mayor que las del grupo de control $(p \leq 0.5)$. Esta información se puede ver con más detalle en la Tabla 5. 
El castellano de la comunidad shipibo de Cantagallo: estudio acústico de la... Paloma Pinillos Chávez

Tabla 5. Subgrupos de bilingües shipibo-castellano y duraciones vocálicas comparadas (valor p) con el grupo control

\begin{tabular}{|l|l|l|l|l|l}
\hline \multicolumn{5}{|c}{ Subgrupo 1 } \\
\hline Vocales & {$[\mathrm{a}]$} & {$[\mathrm{e}]$} & {$[\mathrm{o}]$} & {$[\mathrm{i}]$} & {$[\mathrm{u}]$} \\
\hline $\mathrm{P}(28)$ & 54.84 & 49.22 & 48.93 & 47.51 & 44.42 \\
\hline Grupo control & 54.48 & 45.05 & 50.81 & 49.21 & 50.45 \\
\hline valor $p$ & 0.9384 & 0.5804 & 0.5458 & 0.8619 & 0.2996 \\
\hline \multicolumn{5}{|c|}{ Subgrupo 2 } \\
\hline $\mathrm{P}(18)$ & 64.4 & 64.7 & 65.5 & 42.04 & 60.31 \\
\hline Grupo control & 54.48 & 45.05 & 50.81 & 49.21 & 50.45 \\
\hline valor p & 0.0636 & 0.0237 & 0.0018 & 0.3757 & 0.0908 \\
\hline $\mathrm{P}(29)$ & 59.9 & 59.9 & 54.57 & 52.61 & 50.77 \\
\hline Grupo control & 54.48 & 45.05 & 50.81 & 49.21 & 50.45 \\
\hline valor p & 0.2839 & 0.0427 & 0.4753 & 0.5329 & 0.9436 \\
\hline & & \multicolumn{2}{|c}{ Subgrupo 3 } & & \\
\hline $\mathrm{P}(32)$ & 70.75 & 82.23 & 73.7 & 64.17 & 66.52 \\
\hline Grupo control & 54.48 & 45.05 & 50.81 & 49.21 & 50.45 \\
\hline valor $p$ & 0.0028 & 0.0002 & 0.0001 & 0.0265 & 0.0039 \\
\hline $\mathrm{P}(33)$ & 71.09 & 73.53 & 76.46 & 79.03 & 82.9 \\
\hline Grupo control & 54.48 & 45.05 & 50.81 & 49.21 & 50.45 \\
\hline valor $p$ & 0.001 & 0.0011 & 0.0001 & 0.0003 & 0.0001 \\
\hline $\mathrm{P}(61)$ & 72.64 & 89.91 & 70.9 & 68.5 & 68.74 \\
\hline Grupo control & 54.48 & 45.05 & 50.81 & 49.21 & 50.45 \\
\hline valor p & 0.0011 & 0.0001 & 0.0001 & 0.012 & 0.0019 \\
\hline & & & & &
\end{tabular}

Además, vinculé los resultados de estos subgrupos con los resultados del BLP. A partir del continuo de bilingüismo que se observa en la Tabla 6, se puede observar que el participante $\mathrm{P}(28)$ del subgrupo uno (duración vocálica igual a la del grupo control) tiene puntaje de BLP negativo, es decir, mayor dominancia de shipibo. Por otro lado, $\mathrm{P}(18)$ y $\mathrm{P}(29)$ del subgrupo dos (duración de al menos una vocal mayor a la del grupo control) tienen los puntajes extremos del continuo. $\mathrm{P}(18)$ tiene el único puntaje positivo, dominancia hacia el castellano, mientras que $\mathrm{P}(29)$ tiene el puntaje negativo más alto de todos. Por último, $\mathrm{P}(32), \mathrm{P}(33)$ y $\mathrm{P}(61)$, el subgrupo 
El castellano de la comunidad shipibo de Cantagallo: estudio acústico de la...

Paloma Pinillos Chávez

tres (duración vocálica mayor a la del grupo control) tienen puntajes negativos, lo cual indica su dominancia hacia el shipibo.

Tabla 6. Subgrupos de bilingües shipibo-castellano y duraciones vocálicas ubicados en un continuo según su puntaje de BLP

\begin{tabular}{ll|l|l|l|l|l}
\hline $\begin{array}{l}\text { Puntaje del } \\
\text { BLP }\end{array}$ & 52.766 & -40.5 & -49.85 & -61.384 & -65.924 & -94.62 \\
\hline Participante & $\mathrm{P}(18)$ & $\mathrm{P}(28)$ & $\mathrm{P}(32)$ & $\mathrm{P}(33)$ & $\mathrm{P}(61)$ & $\mathrm{P}(29)$ \\
\hline División & $\begin{array}{l}\text { Subgrupo } \\
2\end{array}$ & $\begin{array}{l}\text { Subgrupo } \\
1\end{array}$ & $\begin{array}{l}\text { Subgrupo } \\
3\end{array}$ & $\begin{array}{l}\text { Subgrupo } \\
3\end{array}$ & $\begin{array}{l}\text { Subgrupo } \\
3\end{array}$ & $\begin{array}{l}\text { Subgrupo } \\
2\end{array}$ \\
\hline
\end{tabular}

\section{Discusión}

Respondiendo a la primera pregunta (¿Los patrones de duración del shipibo han influenciado la duración vocálica del castellano de los hablantes bilingües shipibo-castellano?), los resultados de los datos de este estudio sugieren que hay una influencia del patrón de duración vocálica del shipibo en la duración de las vocales castellanas de los hablantes bilingües shipibo-castellano. Como vimos en los resultados, el promedio total de las vocales castellanas de estos hablantes bilingües es significativamente mayor a la de los hablantes monolingües de castellano, hecho que se puede explicar a partir de la noción del tamaño mínimo de palabra. Estos datos parecen indicar que, así como en shipibo, las vocales del castellano de estos bilingües también requieren de un mínimo de dos moras cuando la palabra es un monosílabo con el fin de cumplir con el peso silábico correspondiente. De esta forma, producir vocales con una mora (menor duración) en monosílabos infringirá la regla del tamaño mínimo de palabra.

Este argumento se fortalece con los resultados de la duración vocálica de los paradigmas de los monosílabos nominales. Así como las vocales del shipibo, las vocales castellanas también presentan mayor duración en las palabras derivadas de monosílabos nominales, duración que es virtualmente igual a la de las vocales de los monosílabos. Esta característica se puede explicar a partir de la herencia de la duración vocálica de monosílabos nominales a sus respectivos paradigmas. Como explica Elías-Ulloa (2011), en shipibo los paradigmas de los monosílabos nominales heredan este patrón de duración y, a partir de los resultados, podemos ver que este patrón se ha transferido a las vocales del castellano en estos bilingües. 
El castellano de la comunidad shipibo de Cantagallo: estudio acústico de la...

Paloma Pinillos Chávez

Asimismo, los resultados también permiten descartar que una mayor duración vocálica es un patrón general del castellano hablado por estos bilingües. Esto se sustenta con las duraciones vocálicas de los bisílabos de estos hablantes, ya que su duración fue significativamente igual a la de los monosílabos del grupo de monolingües y significativamente menor a la de las vocales de los monosílabos de los bilingües.

En cuanto a la segunda pregunta de investigación (¿La duración vocálica de estos hablantes bilingües varía dependiendo de su nivel de bilingüismo?), los datos indican que hay una tendencia a que los hablantes con mayor dominancia de shipibo produzcan vocales con mayor duración, sobre todo si nos enfocamos en el subgrupo tres. En base a estos datos, se podría sugerir que una mayor duración en la vocal está relacionada con el dominio del shipibo. Sin embargo, basada solo en estos resultados, es difícil asociar una menor duración vocálica con un menor dominio en shipibo. Si analizamos a cada participante de los otros subgrupos individualmente, vemos que la participante $\mathrm{P}(18)$ es la más joven de esta muestra y es la única educada íntegramente en castellano, por lo que se explica su puntuación positiva en el BLP. Sin embargo, es parte del subgrupo dos ya que dos de sus cinco vocales son producidas con una duración significativamente mayor a la del grupo control. Por otro lado, las vocales de los participantes $\mathrm{P}(28)$ y $\mathrm{P}(29)$ no presentan el patrón de duración esperado. Por un lado, $\mathrm{P}(28)$ produce vocales con la misma duración que las del grupo control, mientras que $\mathrm{P}(29)$ solo produce una de las cinco vocales con mayor duración que las del grupo control. Ahora bien, si bien la duración de las vocales de estos dos participantes es más cercana a la del grupo control que a la del subgrupo tres (duración vocálica significativamente mayor a la del grupo control), ambos participantes muestran mayor dominancia del shipibo según los resultados del BLP. Inclusive, el participante $\mathrm{P}(29)$ es el que mayor puntaje negativo obtuvo, lo que lo coloca como el más dominante en shipibo. Así, teóricamente se esperaría que estos dos bilingües, al ser dominantes en shipibo en esta muestra, presenten una duración vocálica significativamente mayor en los contextos esperados, sin embargo, esto no se dio.

La pregunta acá es qué está sucediendo en el caso de estos tres participantes, $\mathrm{P}(18), \mathrm{P}(28)$ y $\mathrm{P}(29)$, ya que la duración de sus vocales castellanas no se correlaciona con su nivel de bilingüismo. Para tener una mejor idea sobre estos casos, revisé las respuestas que estos tres participantes dieron en sus cuestionarios del BLP, pero ya no como un puntaje total, sino por secciones. Al revisar las respuestas dadas en las cuatro secciones de estos cuestionarios (historial lingüístico, uso de

184 Lengua y Sociedad. Revista de Lingüística Teórica y Aplicada 
la lengua, dominio de la lengua y actitudes lingüísticas), encontré información relevante sobre su identidad cultural y competencia lingüística hacia el shipibo y el castellano.

En el caso de la sección de competencia lingüística, la participante $\mathrm{P}(18)$, se calificó como más dominante en castellano que en shipibo. Calificó su dominio al hablar, entender, leer y escribir el castellano significantemente mayor al del shipibo. En cuanto a su actitud lingüística, indicó que tiene una actitud más positiva hacia el castellano que hacia el shipibo. Señaló que se siente más como «sí misma» e identificada con la cultura hispanohablante que con la cultura shipibohablante. Además, indicó que es más importante para ella usar el castellano como hablante nativo y que otros piensen que es hablante nativo de esta lengua, mas no señaló esta relación con el shipibo. En el caso de los participantes $\mathrm{P}(28)$ y $\mathrm{P}(29)$, se calificaron igual de dominantes en castellano y en shipibo tanto al hablar, entender, leer y escribir. Ahora bien, en cuanto a sus actitudes lingüísticas, ambos mostraron una actitud significativamente más positiva hacia el shipibo que hacia el castellano. Ambos se sienten como «sí mismos» al usar shipibo mas no al usar castellano. Igualmente, para ambos es más importante que otros los reconozcan como hablantes nativos de shipibo mas no como hablantes nativos de castellano.

Es interesante ver en estos tres casos cómo la duración vocálica es opuesta a lo esperado a partir de la noción del nivel de bilingüismo. Tenemos un caso de transferencia de la duración en una participante que se auto asigna como no dominante del shipibo y cuya actitud lingüística hacia esta lengua no es positiva. Por otro lado, tenemos dos casos en los que esta transferencia de la duración vocálica es mínima o nula, sin embargo, los participantes muestran una actitud lingüística positiva hacia el shipibo y valoran el dominio lingüístico que tienen sobre esta lengua. Si bien los datos en este estudio se concentran en un grupo reducido de participantes bilingües, estos resultados son de interés para abrir más puertas a una investigación más profunda y detallada sobre las posibles correlaciones que existen entre actitudes lingüísticas, concepción de dominio de la lengua y performance lingüístico. Además, tenemos que considerar que estos tres participantes son los más jóvenes de la muestra y los que mayor contacto tienen con el exterior de la comunidad. Este punto también resulta de interés para explorar más a fondo la percepción y valoración que las generaciones más jóvenes tienen del shipibo. 
El castellano de la comunidad shipibo de Cantagallo: estudio acústico de la...

\section{Conclusiones}

Los resultados en la muestra estudiada indican que los hablantes bilingües shipibo-castellano de la comunidad de Cantagallo producen vocales con una duración significativamente mayor a las vocales producidas por hablantes monolingües de castellano. Esta característica puede explicarse a partir de la transferencia del requerimiento del peso silábico del shipibo al castellano. A partir de esta noción, las vocales castellanas de estos hablantes bilingües presentarían mayor duración pues tienen un requerimiento de dos moras en monosílabos nominales y en sus respectivos paradigmas. A partir del nivel de bilingüismo de estos hablantes, los resultados muestran, por un lado, que participantes que muestran mayor dominancia del shipibo también presentan mayor duración vocálica. No obstante, la relación de una mayor duración en la vocal con el dominio del shipibo no se presenta en todos los participantes. Estos últimos resultados llevan a la discusión de las actitudes lingüísticas de los jóvenes bilingües de esta comunidad hacia el shipibo, por lo que se sugiere un estudio más profundo sobre la percepción y valoración que se le da a esta lengua.

\section{Referencias bibliográficas}

Abramson, A. S., y Reo, N. (1990). Distinctive Vowel Length-Duration vs. Spectrum in Thai. Journal of Phonetics, 18(2), 79-92.

Adelaar, W. F., Valenzuela, P., y Zariquiey, R. (2011). Estudios sobre lenguas andinas y amazónicas: homenaje a Rodolfo Cerrón-Palomino. Pontificia Universidad Católica del Perú.

Amengual, M. (2011). Spanish and Catalan in Majorca: Are There ContactInduced Changes in the Catalan Vowel System? En Ortiz-López (Ed.), Selected proceedings of the 13th Hispanic linguistics symposium (pp. 214-223). Cascadilla Proceedings Project.

Amengual, M. (2016). The perception and production of language-specific mid-vowel contrasts: Shifting the focus to the bilingual individual in early language input conditions. International Journal of Bilingualism, 20(2), 133-152.

Anzualdo, J. (2015). Cartografiando la historia: desarrollo demográfico de la comunidad de Cantagallo. Trabajo presentado en el XI Congreso Nacional de Geografía «Ing. Pablo Sánchez Zevallos». Geografía, desarrollo y sostenibilidad territorial: enfoques, políticas y estrategias, Cajamarca, Perú. 
Birdsong, D., Gertken, L. M. y Amengual, M. (2012, 20 de enero). Bilingual Language Profile: An Easy-to-Use Instrument to Assess Bilingualism [Archivo de video]. COERLL, University of Texas at Austin. https://sites.la.utexas.edu/ bilingual

Boersma, P. y Weenink, D. (2015). Praat: doing phonetics by computer (Versión 5.4.06) [Software de computación]. http://www.praat.org

Chládková, K., Escudero, P. y Boersma, P. (2011). Context-specific Acoustic Differences Between Peruvian and Iberian Spanish Vowels. Journal of Acoustical Society of America, 13o(1), 416-428.

Elias-Ulloa, J. (2006). Theoretical aspects of Panoan metrical phonology: disyllabic footing and contextual syllable weight. PhD diss., Rutgers University.

Elías-Ulloa, J. (2011). Una documentación acústica de la lengua Shipibo-Conibo (Pano): con un bosquejo fonológico.

García, M. (2016). Sobre la duración vocálica y la entonación en el español amazónico peruano. Lengua y Sociedad, 14(2), 5-29.

Guion, S. G. (2003). The vowel systems of Quichua-Spanish bilinguals. Phonetica, $60(2), 98-128$.

Instituto Nacional de Estadística e Informática (INEI). (2007). II Censo de Comunidades Indígenas de la Amazonía Peruana 2007. Resultados definitivos.

Ministerio de Cultura del Perú. (2019). Base de Datos de Pueblos Indígenas y Originarios. http://bdpi.cultura.gob.pe/pueblo/shipibo-konibo

Morrison, G. y Escudero, P. (2007). A cross-dialectal comparison of Peninsular-and Peruvian- Spanish Vowels. En Trouvain, J. y Barry, W. (Eds.), Proceedings from the XVI International Congress of Phonetic Sciences (pp. 1505-1508). Saarbrücken.

Navarro Tomás, T. (1916). Cantidad de vocales acentuadas. Revista de Filología Española, 3, 387-407.

Navarro Tomás, T. (1917). Cantidad de vocales inacentuadas. Revista de Filología Española, 4, 371-388.

O'Rourke, E. (2010). Dialect differences and the bilingual vowel space in Peruvian Spanish. En M. Ortega-Llebaria (Ed.), Selected Proceedings of the 4 th Conference on Laboratory Approaches to Spanish Phonology (pp. 20-30). Cascadilla Proceedings Project.

Quilis, A. y Esqueva, P (1983). Realización de los fonemas vocálicos españoles en posición fonética normal. En M. Esgueva y M. Cantarero (Eds.), Estudios de Fonética I (pp. 159-252). Consejo Superior de Investigaciones Científicas. 
El castellano de la comunidad shipibo de Cantagallo: estudio acústico de la...

Paloma Pinillos Chávez

Saldaña Fernández, C. (2009). El ritmo acentual del castellano amazónico. En J. Calvo Pérez y L. Miranda Esquerre (Eds.), Palabras fuera del nido: vertientes sincrónica y diacrónica del español en contacto (pp. 301-309). Universidad de San Martín de Porres.

\section{Trayectoria académica de la autora}

Paloma Pinillos Chávez es candidata a doctora en Lingüística Hispánica por The Ohio State University (osu), magíster en Lingüística Hispánica por la Pontificia Universidad Católica del Perú (PUCP) y en Fonética y Fonología por el Consejo Superior de Investigaciones Científicas de Madrid, bachiller y licenciada en Lingüística Hispánica por la PUCP, docente e investigadora en The Ohio State University. Sus campos de investigación son fonética y fonología, español en contacto, bilingüismo, español de herencia y español como segunda lengua en los Estados Unidos. Es embajadora de los cursos de español de herencia en osu y asistente de dirección y contenido del grupo de perfomance de hablantes de español de herencia Performancerus. Colabora en la Comisión de Relaciones Hispanas/ Latinas de Ohio con traducciones inglés-español de documentos oficiales sobre la vacunación del Covid-19 para el Departamento de Salud de Ohio. Es parte de los proyectos Documenting of Latina/o/xs in Ohio stories during COVID-19 through performed storytelling $y$ Social vulnerability, sociolinguistic environment, and COVID-19 information among Spanish-speakers in Columbus, Ohio. Es docente del certificado The Community Interpreter ${ }^{\circledast}$ (TCI): Medical Focus emitido por The Center Languages, Literatures and Cultures de osu.

188 Lengua y Sociedad. Revista de Lingüística Teórica y Aplicada 